\title{
A method for plaiting polymer fibre around natural yarn to form composite fabric
}

\author{
T. Izumi ${ }^{1}$, T. Matsuoka ${ }^{2}$, T. Hirayama ${ }^{2}$, H. Fujita ${ }^{3}$, \\ Y. Miyata ${ }^{4} \&$ K. Fujii ${ }^{5}$ \\ ${ }^{I}$ Graduate School of Mechanical Engineering, \\ Doshisha University, Japan \\ ${ }^{2}$ Department of Mechanical Engineering, Doshisha University, Japan \\ ${ }^{3}$ Hyogo Prefectural Institute of Technology, Japan \\ ${ }^{4}$ Miyata Fuhaku Co. Ltd., Japan \\ ${ }^{5}$ Tohou Co. Ltd., Japan
}

\begin{abstract}
A method has been developed for plaiting polymer fibre around natural yarn using a sawing machine to form a composite fabric. It was tested using polypropylene (PP) fibre and cotton yarn. Investigation of the relationship between the PP weight ratio and the number of PP fibres in the composite fabric revealed that the weight ratio can be easily controlled by adjusting the number of core PP fibres. The developed composite fabric was heat moulded to form fibrereinforced polymer (FRP). Comparison of the stress-strain curve and tensile strength of this FRP with those of FRP made with conventional fabric revealed that, although the tensile strength of the FRP formed using the composite fabric was smaller than that of the conventional FRP, the strain of the developed FRP was larger because of delamination at the PP-cotton yarn interface. The developed FRP was improved by covering its top and bottom surfaces with a thin PP sheet before heat moulding. This prevented stress concentration, resulting in a $30 \%$ increase in tensile strength. Use of this FRP formed material using composite fabric is an effective approach to forming composite materials having high strain performance and tensile strength.
\end{abstract}

Keywords: fibre-reinforced composites, composite yarn, polypropylene, cotton, static tensile test, stress-strain curve. 


\section{Introduction}

Materials made of thermoplastic resin and natural fibres have attracted much attention as environment-friendly materials because they can be easily disposed of using only an incinerator. Moreover, fabrics woven using composite yarn made of natural yarn and resin fibre should have higher strength. However, the methods proposed so far for forming composite yarns (fusion, powder, solvent, and so on [1]) are not cost effective [2, 3]. There is thus a need for novel materials with high recyclability and easy disposability.

Several kinds of natural fibres have been used as a reinforcing material in the quest for better composites [4, 5]. Cotton yarn is particularly attractive because it has higher heat resistance than other fibres such as jute, hemp, and bamboo. High heat resistance is especially important for composites made using thermoplastic resin because they are fabricated at high temperature. While composite fabric has been shown to have sufficient strength for practical application, impregnation of the resin into the fabric is problematic, especially for thermoplastic resin with high viscosity.

We have developed a method for plaiting polymer fibre around natural yarn using a sawing machine to form a composite fabric. We tested it using polypropylene (PP) fibre and cotton yarn. We first investigated the relationship between the PP weight ratio and the number of PP fibres in composite fabric and found that the weight ratio can be easily controlled by adjusting the number of core PP fibres. We then formed fibre-reinforced polymer (FRP) using the composite fabric and heat moulding. Examination of its stress-strain curve and tensile strength with those of FRP made with conventional fabric revealed that, although the tensile strength of the FRP formed using the composite fabric was smaller than that of the conventional FRP, its strain was larger because of delamination at the PP-cotton yarn interface. Covering the top and bottom surfaces of the laminated composite fabric with a thin PP sheet before heat moulding reduced the stress concentration, resulting in a $30 \%$ increase in tensile strength.

Use of this plaiting method should be easy to implement because it requires only the addition of a small sawing machine. Moreover, the moulding conditions are easy to control because the amount of PP fibre can be easily adjusted by changing the plaiting pitch and the number of PP fibres.

\section{Fabrication and structure of composite yarn and composite fabric}

\subsection{Composite yarn}

The structural model of the composite yarn we developed is shown in Figure 1(a). To increase the matrix polymer content, some matrix fibres, the 'core matrix fibres', are arranged in parallel along the reinforcing yarn, as shown in Figure 1(b). The matrix fibres are plaited around the centre of the reinforcing yarns, as illustrated in Figure 1(c), by using a mellow sewing machine. The 
twisted cotton yarn, made with four tenth-string single yarns was used as the reinforcement yarn at the centre. We used monofilament PP fibres (300D) as core fibres. Their mechanical properties are listed in Table 1. The fibre diameter, plating pitch $L$, and the number of reinforcing PP fibres can be freely selected, meaning that the composite moulding conditions are easy to control. A photograph of the composite yarn is shown in Figure 1(d): the left shows the composite yarn with only cotton yarn at the centre; the right shows the yarn with three core PP fibres at the centre along a cotton yarn.

Table 1: $\quad$ Mechanical properties of PP fibre (300D).

\begin{tabular}{c|c|c|c|c|c|c|c}
\hline \multirow{2}{*}{ Density } & \multicolumn{2}{|c|}{ Bend } & \multicolumn{3}{c|}{ Pull } & $\begin{array}{c}\text { Deflection } \\
\text { temperature } \\
\text { under load }\end{array}$ & $\begin{array}{c}\text { Rockwell } \\
\text { hardness }\end{array}$ \\
\cline { 2 - 7 } & Modulus & Strength & Modulus & Yield stress & Stress & \\
\hline $0.9\left[\mathrm{~g} / \mathrm{cm}^{3}\right]$ & $1500[\mathrm{MPa}]$ & $43[\mathrm{MPa}]$ & $1500[\mathrm{MPa}]$ & $34[\mathrm{MPa}]$ & $100[\mathrm{Mpa}]$ & $100\left[{ }^{\circ} \mathrm{C}\right]$ & 100 \\
\hline \hline
\end{tabular}

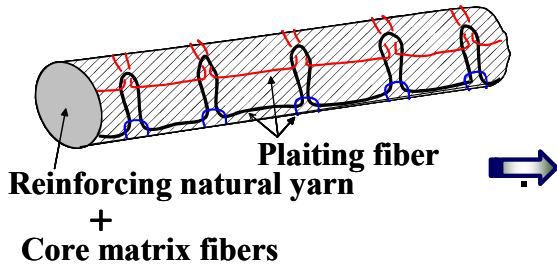

(a) Structural model of composite yarn

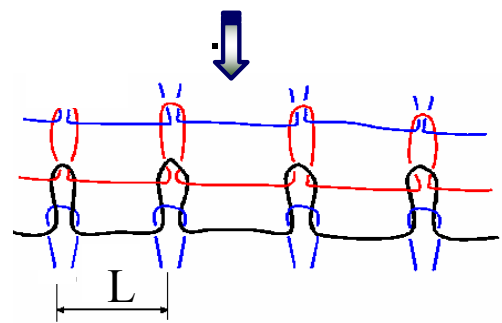

(c) Configuration of reinforcing yarn

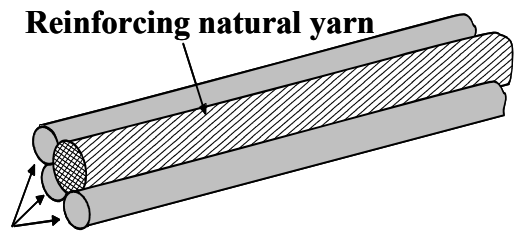

Core matrix fibers

(b) Configuration of plaiting fibre

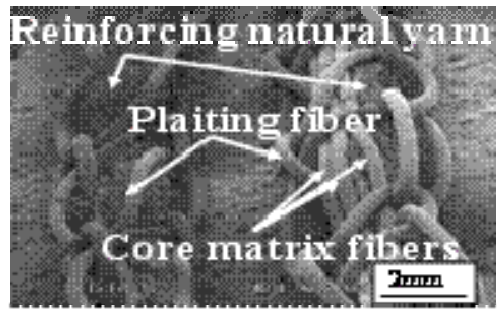

(d) SEM image and core matrix fibres

Figure 1: Composite yarn.

To investigate the relationship between the weight ratio of the PP and the number of core PP fibres, we prepared five composite yarn samples with the number of core PP fibres ranging from one to five. The plaiting pitch was set to $3.9 \mathrm{~mm}$. The weight ratio of the PP in the composite yarn was measured by dissolving the cotton yarn using $70 \%$ sulphuric acid. The change in the weight ratio with the number of core PP fibres is shown in Table 2. 
Table 2: Weight ratio of PP and cotton.

\begin{tabular}{c|c|c|c|c|c}
\hline \hline The number of core PP fibers & 1 & 2 & 3 & 4 & 5 \\
\hline Cotton $[\mathrm{wt} \%]$ & 49.95 & 45.79 & 41.85 & 38.96 & 36.89 \\
\hline PP $[\mathrm{wt} \%]$ & 50.05 & 54.21 & 58.15 & 61.04 & 63.11 \\
\hline
\end{tabular}

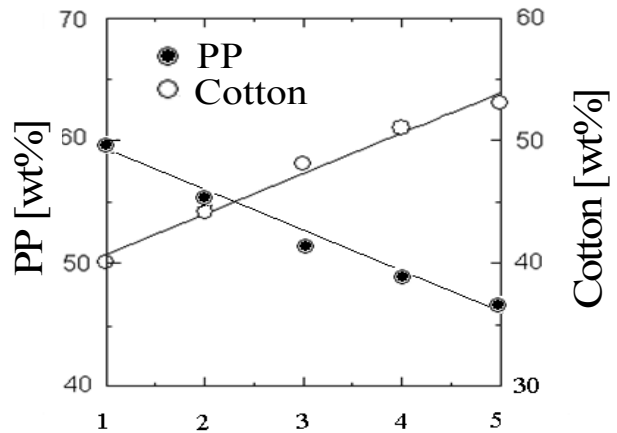

Figure 2: $\quad$ The number of core fibres.

As shown in Figure 2, the number of core PP fibres and the PP weight ratio had an almost in linear relationship. This means that the weight ratio can be easily controlled by adjusting the number of core PP fibres.

The composite yarn fabricated using our plating method has four key features.

* Different types of reinforcement yarns and plaiting fibres can be used.

* The matrix content can be easily adjusted due to the easy adjustability of the plaiting pitch, the number of core matrix fibres, and so on.

* Composite fabrics with various weave patterns (plain, braid, and 3D) can be easily fabricated.

* Composite fabrics woven using the developed composite yarn should have high strength and good flexibility, making them suitable for various applications.

\subsection{Composite fabric}

Composite fabric was fabricated by weaving the composite yarn using a weaving machine. The number of core PP fibres was 1,3 , or 5 , the weave pattern was plain, and the warp and woof were respectively set to 12 and 11 per inch. The thickness was $0.8 \mathrm{~mm}$ for the fabric woven with cotton yarn only and $1.5 \mathrm{~mm}$ for fabric woven using the composite yarn.

\section{Moulding method for FRP plate}

To form a fibre-reinforced plastic (FRP) plate, three composite fabrics were stacked in a laminate structure in a mould die and heated with a hot press. Lubricant was previously coated on the top and bottom surfaces of the die to prevent the PP from adhering to it. The moulding temperature, pressure, and time 
were set to $190^{\circ} \mathrm{C}, 1.5 \mathrm{MPa}$, and 7 minutes. After heating, the die was kept at room temperature for ten minutes.

To compare the mechanical properties between the developed fabric FRP and conventional fabric FRP, we prepared fabric FRP using cotton fabrics and thin PP sheets. The cotton fabrics were arranged in a plain weave pattern, and the PP sheets were stacked in a laminate structure, as illustrated in Figure 3. The sheets were $0.11,0.16$, and $0.2 \mathrm{~mm}$ thick. The FRPs made using the developed fabric are identified as P1, P2, and P3, as listed in Table 3. Those made using the conventional fabric and a PP sheet are identified as S1, S2, and S3, as listed in Table 4. For instance, test specimens S1 and P1 were moulded using the same PP weight ratio. The FRP plates were all $240 \times 240 \mathrm{~mm}$. The moulding conditions were the same for both types of FRP.

Table 3: $\quad$ Identification of FRPs formed using composite yarn.

\begin{tabular}{c|c}
\hline \hline Test specimens & The number of core PP fibers \\
\hline P1 & 1 \\
\hline P2 & 3 \\
\hline P3 & 5 \\
\hline
\end{tabular}

Table 4: Identification of FRPs formed using PP sheet and conventional fabric.

\begin{tabular}{c|c}
\hline \hline Test specimens & Thickness of PP sheet $[\mathrm{mm}]$ \\
\hline S1 & 0.11 \\
\hline S2 & 0.16 \\
\hline S3 & 0.2 \\
\hline
\end{tabular}

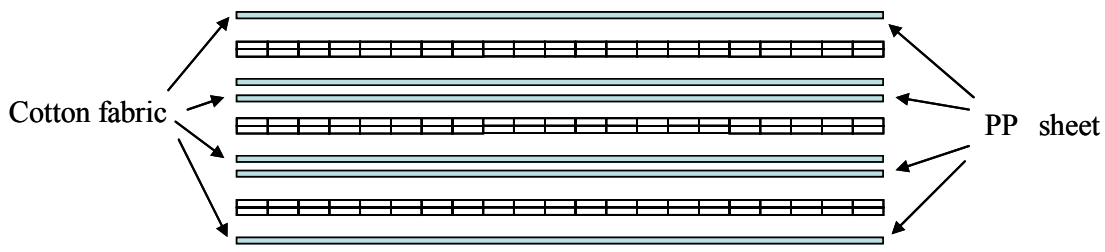

Figure 3: Construction of conventional fabric FRP.

\section{Static tensile test}

\subsection{Specimens}

The FRP plates were cut into specimens $25 \mathrm{~mm}$ (12 warp) in width and $200 \mathrm{~mm}$ in length with a diamond cutter for static tensile testing. Aluminium tabs, $50 \times$ $25 \mathrm{~mm}$ ) were attached to both ends of each specimen, as shown in Figure 4. 


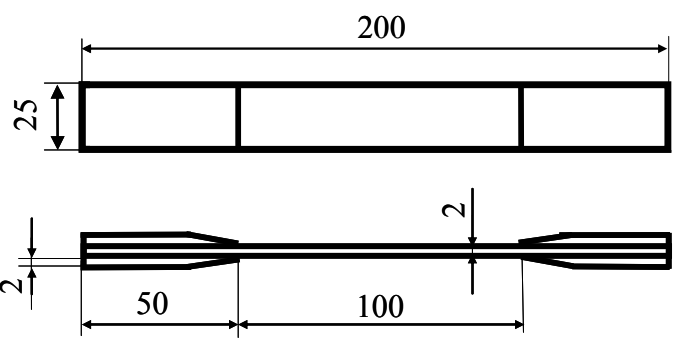

Figure 4: $\quad$ Test specimens for tensile test.

\subsection{Conditions}

An automatic tensile tester with displacement control was used. The tensile speed was set to $5 \mathrm{~mm} / \mathrm{min}$. The room temperature and humidity were $20^{\circ} \mathrm{C}$ and $65 \%$. The number of test samples was six for each specimen. The fracture faces after the tensile test were observed using a photon microscope (VQ-Z50, Keyence).

\section{Results and discussion}

Figure 5 show the tensile stress-strain curves for specimens P1, P2, P3, S1, S2, and S3, and Figure 6 shows the tensile strength for each specimen.

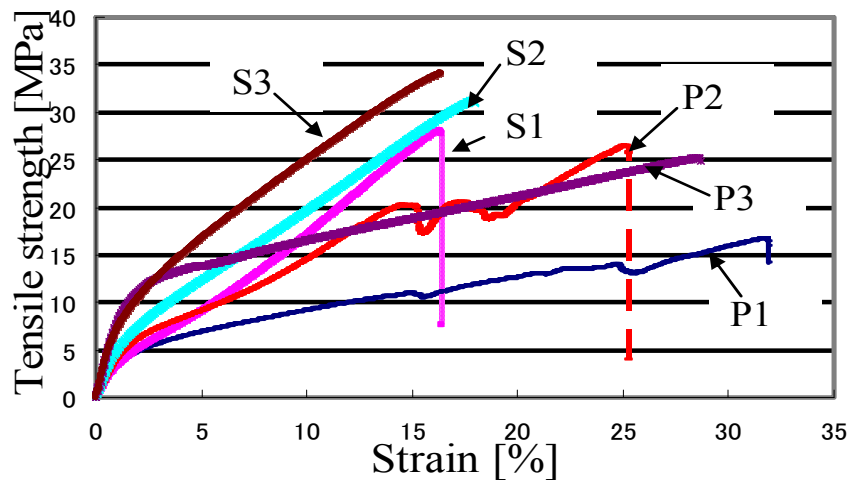

Figure 5: $\quad$ Tensile stress-strain curves for each FRP specimen.

As shown in Figure 5, the tensile strain for P2 was about 15-20\% low. Such a decrease for S2 is not evident. As shown in Figure 6, the tensile strength increased with the PP resin content. Since the cotton yarn content was the highest in P1 and S1, P1, S1, P2, and S2 had insufficient resin content to have a noticeable composite effect.

SEM photographs of a cross-section of P2 and S2 at a point where the strain was $15-20 \%$ are shown in Figure 7 . With the P2 specimen, delamination 


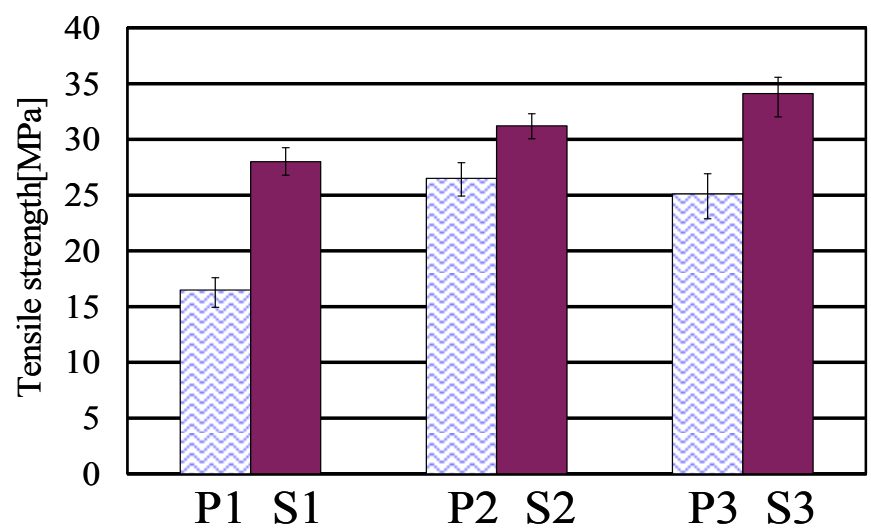

Figure 6: Tensile strength for each FRP specimen.

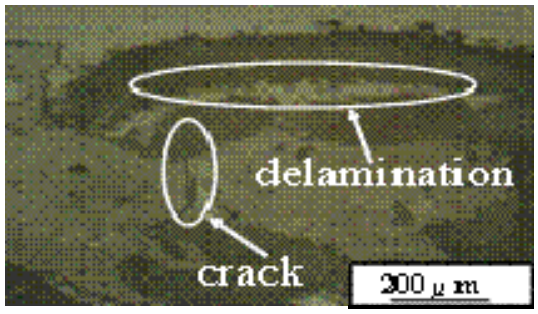

(a)

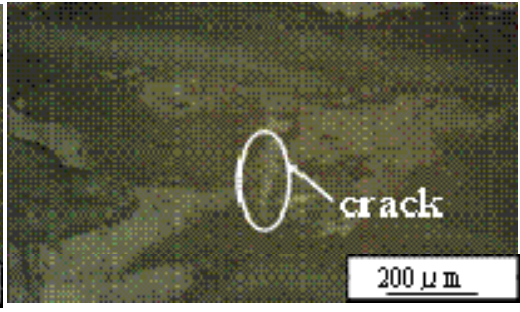

(b)

Figure 7: $\quad$ SEM images of the FRPs in cross-section after tensile test. (a) P2 specimen, developed FRP with composite yarn. (b) S2 specimen, conventional FRP with cotton fabric and PP sheet.

occurred at the boundary between the PP and cotton yarn. As a result, although the tensile strength of the developed FRP was lower than that of the conventional FRP, its maximum strain was larger. The developed FRP thus has better strain performance than the conventional FRP. The tensile stress was lower in the P3 specimen. This is because P3 had sufficient resin to have a noticeable composite effect.

We next focused on the unevenness on the FRP surface because the developed FRP had more unevenness than the conventional FRP. The large unevenness on the developed FRP surface was due to the PP not uniformly covering the FRP surface, resulting in stress concentration somewhere near the surface. To increase the maximum tensile strength of the developed FRP, we covered the top and bottom surfaces of the composite fabric in laminate with thin PP sheets before heat moulding. This changed the PP weight ratio, as shown in Table 5 . 
Table 5: Weight ratio of PP and cotton.

\begin{tabular}{c|c|c|c}
\hline \hline The number of core PP fibers & 1 & 3 & 5 \\
\hline Cotton $[\mathrm{wt} \%]$ & 40.05 & 32.42 & 27.78 \\
\hline PP $[\mathrm{wt} \%]$ & 59.95 & 67.58 & 72.22 \\
\hline
\end{tabular}

The stress-strain curves of the improved FRP are shown in Figure 8; the improved FRPs are identified as "PS". For instance, the specimen with PP sheets added to the surfaces of P1 is "PS1". Figure 9 shows the tensile strength of the Pand PS-type specimens. Adding the sheets was effective for reducing the stress concentration, and the tensile strength was improved by about $30 \%$ for PS1.

A check for delamination after tensile testing showed that the FRP formed using the composite yarn had high strain performance. Moreover, adding the PP sheets to the surfaces of the composite fabric before heat moulding effectively improved tensile strength.

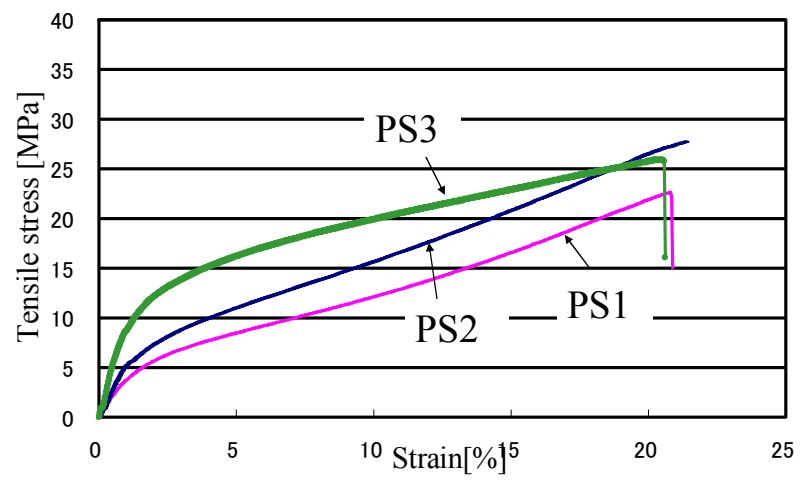

Figure 8: Tensile stress-strain curves of FRP after adding PP sheet to top and bottom surfaces.

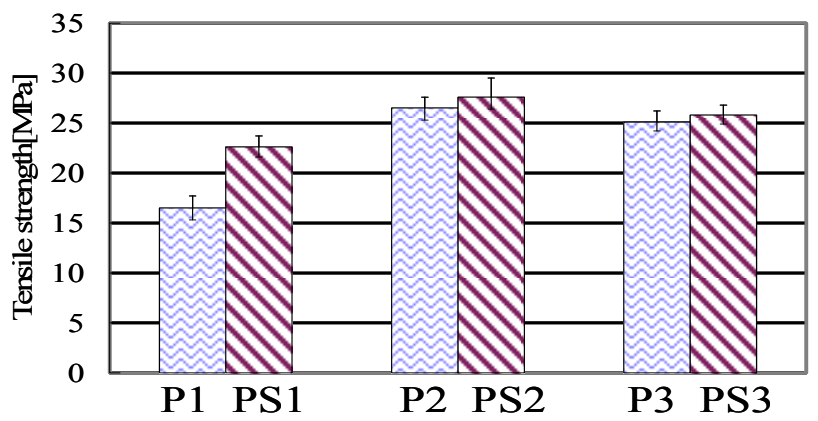

Figure 9: Tensile strength before (P) and after (PS) adding PP sheets to surfaces. 
Future work includes identifying the optimum conditions (number of core PP fibres and pitch of plaiting fibres) for moulding composite materials with high strength.

\section{Main results}

(1) The cotton content in the composite yarn had an almost linear relationship with the number of core PP fibres.

(2) The composite fabric made using cotton/PP composite yarn was flexible.

(3) The strain of the FRP plate made using the composite fabric was larger than that of one made using a PP sheet because using the composite fabric has caused flaking off.

(4) The unevenness of the FRP surface decreased the tensile stress. Reducing the unevenness by using a PP sheet increased the tensile strength about $30 \%$ for the $\mathrm{P} 3$ specimens.

\section{Acknowledgements}

This study was supported by the High Technological Research Project of Doshisha University and by grants from the Ministry of Education, Culture, Sports, Science and Technology, Japan.

\section{References}

[1] Hong H, Araujo M, Fangueiro R. 3D Technical Fabrics. Knit Int. 1996:1232:55-7.

[2] Buck M., Phoenix TPC Inc, "Continuous Fiber Thermoplastic Composites Materials \& Processing Technologies", Proc. of 10th Japan International SAMPE Symposium \& Exhibition 2007 (2007).

[3] Larbig H, Scherzer H, Dahlke B, Poltrock R. Natural fibre reinforced foams based on renewable resources for automotive interior applications. Journal of Cellular Plastics 1998:34 (July/August):361-79.

[4] Leao A, Rowell R, Tavares N. Applications of natural fibres in automotive industry in Brazil-thermoforming process. Proceedings of the 4th International Conference on Frontiers of Polymers and Advanced Materials. Cairo, Egypt: Plenum Press; 1997. pp. 755-60.

[5] J. Muzzy, Varughese B., Thammongkol V. and Tincher W. "Electrostatic Prepregging of Thermoplastic Matrices", SAMPE Journal, Vol. 25, No. 5, pp. 15-21 (1989). 